\title{
Caracterización del gasto de bolsillo en salud en Chile: una mirada a dos sistemas de protección
}

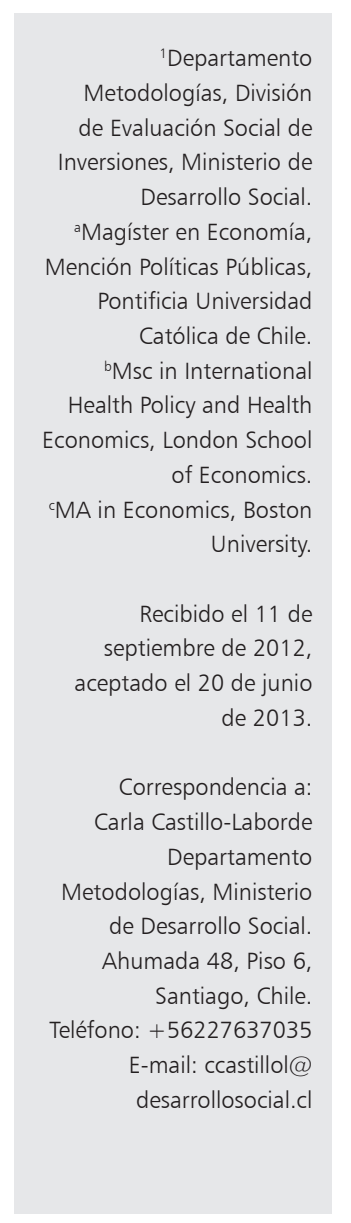

\author{
CARLA CASTILLO-LABORDE $E^{1, \mathrm{a}, \mathrm{b}}$, PABLO VILLALOBOS DINTRANS ${ }^{\mathrm{a}, \mathrm{c}}$
}

Background: The importance of the Out-of-Pocket payments (OOP) for health, as well as the share of the household's income devoted to this purpose provide relevant information for the characterization of the health system. Chile has two coexisting alternative insurance systems (a public system called FONASA and a private system generically called ISAPRE or preventive health institutions), becoming an interesting scenario for the analysis of $O O P$ and the comparison of the financial protection provided by different insurance schemes. Aim: To characterize OOP for households enrolled in FONASA and ISAPRE. Material and Methods: Using data from the Chilean Household Budget Survey 2007, we calculated measures of OOP payments for households enrolled in FONASA and ISAPRE, as well as different statistics for the items associated to direct health payments and their importance in household's total health expenditure. Results: The average OOP expenditure for FONASA and ISAPRE enrollees in 2007 was about CLP\$ 21.500 and CLP\$ 86.000, respectively (3.8\% and $6.1 \%$ of their income). Drugs were the most important item of this expenditure, ranging from approximately CLP\$ 6.500 for FONASA enrollees (31.4\%) to CLP\$25.800 for ISAPRE enrollees (29.9\%). The poorest ISAPRE households (comparable to some FONASA households) are those with the higher OOP/income ratio. Conclusions: ISAPRE enrollees have larger OOP payments than FONASA enrollees, not only in absolute terms, but also in terms of the share of their income devoted to direct payments.

(Rev Med Chile 2013; 141: 1456-1463)

Key words: Chile; Health care disparities; Health Services administration; Uncompensated care.

S egún el Reporte Mundial de la Salud del año $2000^{1}$, el financiamiento es una de las cuatro funciones de todo sistema de salud, siendo la generación de recursos, la provisión de servicios y la administración las otras tres funciones restantes. Entre las funciones relacionadas con el financiamiento están la recolección de los fondos, la mancomunación de los fondos pre-pagados (pooling) y la compra de servicios². Dependiendo de cómo el sistema de salud aborda su función de financiamiento, y principalmente la de mancomunación, éste puede lograr que los riesgos asociados a salud y sus consecuentes costos financieros puedan ser enfrentados de manera colectiva, en lugar de que cada individuo deba enfrentarlos separadamente.

Es precisamente la función de mancomunación la que está inversamente relacionada con la dependencia de los pagos directos por parte de las personas para financiar sus necesidades en salud (gasto de bolsillo). De hecho, el Reporte Mundial de la Salud $2010^{3}$ señala, que es fundamental aumentar el pre-pago con mancomunación de 
fondos si se quiere alcanzar la meta de cobertura universal de salud en los países.

Por una parte, la proporción del gasto en salud correspondiente a gasto de bolsillo resulta un indicador importante de tener en consideración al momento de evaluar el desempeño de los sistemas de salud. En Chile, en el año 2009, el gasto de bolsillo representó 34\% del gasto total en salud, el cual resulta considerablemente alto si se compara con el promedio para los países de la Organización para la Cooperación Económica y el Desarrollo (OECD) que ese mismo año ascendió a 19,2\%, con un rango desde 7,3\% y 10,5\% para Francia y Reino Unido respectivamente, hasta $47,8 \%$ para México. Por otra parte, el mismo 34\% no parece tan alto si se compara con el promedio para países sudamericanos, que ese año fue de $31 \%$, con un rango desde $8 \%$ para Colombia, hasta $54 \%$ para Venezuela ${ }^{4}$.

El gasto de bolsillo en salud por parte de los hogares en relación ya sea a su ingreso, gasto total, capacidad de pago o consumo final, suele ser una medida usada para evaluar el desempeño de los sistemas en cuanto a la protección financiera que ofrecen. En el contexto internacional, a nivel de hogares, Chile también presenta una proporción alta de gasto de bolsillo como porcentaje del consumo final de los hogares: mientras el promedio de los países de la OECD es 3,2\%, en el caso chileno, esta cifra alcanza 4,6\%, siendo sólo superado por México, Grecia y Suiza, con 4,7\%, 5,4\% y 6,2\% respectivamente ${ }^{5}$. A partir de los datos que presenta una publicación que compara el gasto de bolsillo a nivel de países latinoamericanos, Chile muestra una proporción de gasto de bolsillo sobre gasto total de $11,6 \%$, el más alto de los 12 países considerados (basados en los resultados de la Encuesta Nacional sobre Satisfacción y Gasto en Salud 2005) .

También en este contexto, los resultados de la Encuesta de Presupuestos Familiares, EPF ${ }^{7}$ muestran que en Chile, 5,4\% del gasto total de los hogares es destinado a salud (como gasto de bolsillo), y que esta proporción es mayor mientras más ricos son los hogares (3,3\% para hogares del quintil 1 y 5,8\% para hogares del quintil 5). A partir de los resultados de la misma encuesta otro artículo estimó la incidencia del gasto catastrófico en salud en Chile (usando un umbral del 30\% de la capacidad de pago), siendo éste aproximadamente de 3,8 y 3,6\% para 1997 y 2007 respectivamente, mostrando que los hogares de los quintiles 4 y 5 son los que presentan mayor probabilidad de caer en gasto catastrófico en salud. Además, los autores muestran que los medicamentos son el componente más importante del gasto de bolsillo (55\% del gasto en 2007), cobrando mayor relevancia aun para los quintiles de menores ingresos (68 y 47\% para quintil 1 y 5 respectivamente) ${ }^{8}$.

Sin embargo, a la fecha no se cuenta con resultados que diferencien el gasto de bolsillo en salud para los beneficiarios de los distintos seguros de salud en nuestro país. Cabe recordar que en Chile, la reforma de la salud del año 1980 cambió la estructura de financiamiento del sistema de salud, permitiendo la introducción de Instituciones de Salud Previsional (Isapre) como fondos alternativos al Fondo Nacional de Salud (Fonasa).

Las diferencias entre ambos tipos de seguros (público y privados con fines de lucro) son múltiples. Por una parte, y aunque las contribuciones de salud son obligatorias para todos los trabajadores asalariados, la forma de contribuir a las Isapre y al Fonasa difiere; mientras la contribución al Fonasa corresponde a $7 \%$ del salario de los asalariados, en las Isapre las primas pueden variar considerablemente entre individuos, siendo como mínimo $7 \%$ del salario, pero ajustadas por riesgo (sexo y edad) y permitiendo incrementos de manera de otorgar mayores coberturas financieras (cotizaciones adicionales). Por otra parte, el Fonasa cuenta con financiamiento a través de impuestos generales, de manera de cubrir a los grupos de menores ingresos (Fonasa grupo A), los cuales no contribuyen financieramente al sistema. Así, otra diferencia importante es precisamente la composición de las carteras de beneficiarios de ambos tipos de fondos: mientras los más ricos y sanos generalmente cotizan en las Isapre, lo más pobres y con más carga de enfermedades lo hacen en el Fonasa.

El año 2011, el 76,2\% de la población chilena estaba cubierta por el Fonasa, y el 16,9\% por las Isapre $^{9}$. Ese mismo año, de acuerdo a los tramos de ingreso (de menor a mayor ingreso), 29,5\% de los beneficiarios de Fonasa pertenecían al tramo A, $31,1 \%$ al tramo B, $16,9 \%$ al tramo C y $22,5 \%$ al tramo D.

En el contexto del sistema de salud chileno y su fragmentación, el objetivo del presente artículo es caracterizar la protección financiera entregada por ambos tipos de fondos a sus beneficiarios, en 
términos del gasto de bolsillo en salud en que éstos deben incurrir.

\section{Material y Método}

Los datos para los análisis fueron obtenidos de la VI Encuesta de Presupuestos Familiares (EPF). La encuesta es desarrollada por el Instituto Nacional de Estadísticas (INE) aproximadamente cada 10 años. Originalmente fue diseñada para identificar los cambios en la estructura de gastos de los hogares chilenos, con el fin de actualizar la canasta de consumo usada para el cálculo del Índice de Precios al Consumidor (IPC).

La encuesta es levantada en un período de 12 meses, de manera de capturar las variaciones estacionales del gasto de los hogares a lo largo del año. La sexta versión de la encuesta fue llevada a cabo entre octubre de 2006 y noviembre de 2007. La base de datos incluye información sobre los ingresos y gastos de los hogares, así como información sobre su composición socio-demográfica. La muestra cubre 10.092 hogares distribuidos en 24 sub-muestras independientes, donde cada submuestra corresponde a un período de 15 días del período de referencia.

Para recolectar la información, el INE pide a cada miembro del hogar (mayor de 15 años) que realice un registro de todos sus gastos personales durante un período de 15 días. Además, se solicita un registro de todos los gastos periódicos del hogar, tomando en cuenta el último período de consumo. Para los gastos no periódicos se consideran dos períodos de recordación: uno de 6 meses para el registro de gastos en turismo, equipo para el deporte, artefactos para el hogar, alfombras y revestimiento de pisos, consultas médicas-dentales y de otros profesionales de la salud, exámenes de laboratorio, radiografías y otros procedimientos extra hospitalarios, y servicios de educación; y un período de recordación de 12 meses para gastos en muebles, artefactos y electrodomésticos para el hogar, computación y accesorios, equipos y artículos para la recreación y comunicación, servicios y materiales para mantención y reparación del hogar, vehículos nuevos y usados, lentes ópticos y aparatos ortopédicos.

En primer lugar, se muestran las estadísticas desagregadas sobre gasto en salud por tipo de gasto, separando la información según tipo de seguro de salud al que pertenece cada uno de los hogares (a pesar de que la afiliación es personal se comprobó que sólo en 46 hogares existían miembros del hogar con afiliaciones distintas. En esos casos se asumió la afiliación del jefe de hogar como la del hogar).

A partir de la encuesta es posible identificar 12 tipos distintos de gasto en salud: i) medicamentos; ii) insumos médicos; iii) lentes ópticos; iv) aparatos ortopédicos; v) consulta médica; vi) intervención médica en operaciones; vii) honorarios de otros profesionales; viii) servicio dental; ix) laboratorio y rayos; $\mathrm{x}$ ) hospitalización; xi) servicio de emergencia móvil y; xii) seguro de salud. Esta información permite hacer una caracterización general de los gastos en salud para personas pertenecientes a los distintos sistemas de financiamiento de salud en el país. El análisis presenta información (en niveles y porcentajes) sobre montos gastados, proporción del gasto total en salud y relación de gasto entre afiliados al sistema público y privado. La información es presentada a nivel desagregado (por tramo de Fonasa y por tipo de Isapre) y agregado (siempre como promedios de gasto por hogar).

Adicionalmente se muestran estadísticas de gasto en salud como proporción del ingreso, separando la información según sistema de financiamiento de los hogares y deciles de ingreso. Al respecto, se optó por usar la proporción del gasto de bolsillo sobre el ingreso total de los hogares, por sobre alternativas como gasto total o capacidad de pago (descontando el gasto en alimentación, como se propone en el reporte de la OMS $2000^{1}$ ). Lo anterior con el fin de dar cuenta de la presión que ejerce el gasto de bolsillo en salud sobre el presupuesto familiar, el cual está acotado por el ingreso, y que puede diferir del gasto total del hogar. En particular, en los casos donde el gasto (ya sea gasto total o gasto descontando el gasto en alimentos) es mayor al ingreso, el gasto de bolsillo en salud como proporción del presupuesto familiar sería menor (usando gasto en lugar de ingreso), pero no estaría tomando en cuenta la carga adicional que estos gastos imponen sobre la deuda familiar. De esta forma, el uso del ingreso como denominador en el cálculo del indicador, asume una medida más conservadora, en el sentido que muestra un indicador mayor, en la medida que los hogares tengan más deudas (para un gasto de bolsillo en salud dado). 
Tabla 1. Gasto promedio mensual en salud según tipo de gasto y sistema de salud (\$ abril de 2007)

\begin{tabular}{|c|c|c|c|c|c|c|c|c|}
\hline $\begin{array}{l}\text { Tipo de gasto/ } \\
\text { Sistema de salud }\end{array}$ & $\begin{array}{c}\text { Fonasa } \\
\mathbf{A}\end{array}$ & $\begin{array}{c}\text { Fonasa } \\
\text { B }\end{array}$ & $\begin{array}{c}\text { Fonasa } \\
\mathrm{C}\end{array}$ & $\begin{array}{c}\text { Fonasa } \\
\text { D }\end{array}$ & $\begin{array}{l}\text { Isapre } \\
\text { abierta }\end{array}$ & $\begin{array}{l}\text { Isapre } \\
\text { cerrada }\end{array}$ & $\begin{array}{l}\text { Capredena/ } \\
\text { Dipreca }\end{array}$ & Otros \\
\hline Total de hogares & 113.230 & 360.068 & 273.922 & 167.606 & 411.706 & 17.377 & 31.875 & 6.025 \\
\hline Medicamentos & $3.780,31$ & $6.156,40$ & $6.554,20$ & $10.300,96$ & $26.148,21$ & $18.478,98$ & $17.646,08$ & $27.990,14$ \\
\hline Insumos médicos & 339,38 & 645,58 & 434,44 & 569,04 & $1.274,68$ & 510,62 & 693,44 & 264,91 \\
\hline Lentes ópticos & $1.233,93$ & $2.103,01$ & $2.380,21$ & $3.724,26$ & $5.629,64$ & $7.944,38$ & $6.144,35$ & $4.300,91$ \\
\hline $\begin{array}{l}\text { Aparatos ortopédicos } \\
\text { y terapéuticos }\end{array}$ & $2.180,75$ & 22,86 & 29,58 & 109,51 & 758,28 & - & $2.082,30$ & $3.168,39$ \\
\hline Consulta médica & 682,89 & $3.083,85$ & $3.205,18$ & $3.979,28$ & $15.358,06$ & $12.910,17$ & $5.099,80$ & $8.041,17$ \\
\hline $\begin{array}{l}\text { Intervención médica } \\
\text { en operaciones }\end{array}$ & - & 4,61 & 33,10 & 110,74 & $1.056,00$ & - & - & - \\
\hline $\begin{array}{l}\text { Honorarios de otros } \\
\text { profesionales }\end{array}$ & - & 271,47 & 117,87 & 606,99 & $1.642,37$ & $1.608,39$ & 103,46 & - \\
\hline Servicio dental & - & $3.490,47$ & $3.005,19$ & $5.242,85$ & $12.306,68$ & $4.308,99$ & $1.567,91$ & - \\
\hline $\begin{array}{l}\text { Análisis y servicio de } \\
\text { laboratorio y rayos }\end{array}$ & - & $1.984,40$ & $1.959,85$ & $2.976,70$ & $6.394,87$ & $13.404,58$ & $3.902,24$ & 422,58 \\
\hline Hospitalización & 681,74 & $2.191,01$ & $3.146,07$ & $6.126,05$ & $14.962,76$ & $21.478,75$ & $3.236,16$ & 806,79 \\
\hline $\begin{array}{l}\text { Servicio de } \\
\text { emergencia móvil }\end{array}$ & 545,07 & 18,28 & - & 23,79 & 327,52 & - & 90,43 & 438,59 \\
\hline Seguro de salud & - & 50,42 & 15,24 & 50,07 & 709,77 & - & $1.456,61$ & - \\
\hline Total & $9.444,08$ & $20.022,38$ & $20.880,93$ & $33.820,23$ & $86.568,84$ & $80.644,85$ & $42.022,78$ & $45.433,49$ \\
\hline
\end{tabular}

\section{Resultados}

Las Tablas 1 y 2 presentan la desagregación del gasto en salud (promedio mensual) para los beneficiarios de cada tipo de seguro, de acuerdo a los grupos por tramo ingreso en el caso del Fonasa y a tipo de Isapre (abierta o cerrada). Todos los montos están expresados en pesos de abril de 2007.

Los datos muestran que un beneficiario perteneciente al grupo A del Fonasa gasta de su bolsillo aproximadamente $\$ 9.500$ mensuales en salud, mientras que un beneficiario del grupo $\mathrm{D}$ gasta alrededor de $\$ 34.000$ y un beneficiario de Isapre (abierta o cerrada), aproximadamente $\$ 80.000$ mensuales. Esta gradiente se mantiene para la mayoría de los ítems considerados. Por ejemplo, en el caso de los medicamentos, el gasto va desde $\$ 3.780$ para Fonasa A $(40,03 \%)$ a $\$ 10.300$ para Fonasa D $(30,46 \%)$ y $\$ 26.148$ para los beneficiarios de Isapre abiertas (30,21\%). Otros ítems de gasto interesante de analizar son el de las consultas médicas, cuyo gasto va desde $\$ 682$ para
Fonasa A $(7,23 \%)$ a $\$ 15.358$ para Isapre abiertas $(17,74 \%)$, y el del gasto en hospitalizaciones, que va desde $\$ 681$ para Fonasa A $(7,22 \%)$ a $\$ 14.962$ para Isapre abiertas $(17,28 \%)$. Por último, en el caso de los servicios dentales, no se registra gasto para los beneficiarios Fonasa A, siendo $\$ 3.490$ en promedio para Fonasa B $(17,43 \%)$ y 12.306 para Isapre abiertas $(14,22 \%)$.

En términos agregados, cuando se considera a todos los beneficiarios de Fonasa e Isapre, las Tablas 3 y 4 muestran que el gasto de bolsillo mensual en salud de un beneficiario Fonasa es en promedio $\$ 21.498$, mientras que para las Isapre sería en promedio $\$ 86.329$, siendo este último cuatro veces el primero. Analizando los montos e importancia relativa de los mismos ítems revisados de manera desagregada, se observa que el gasto promedio en medicamentos para los beneficiarios Fonasa asciende a $\$ 6.740(31,36 \%)$ mientras que para Isapre sería de $\$ 25.837$ (29,93\%). Por otra parte, el gasto promedio en consultas médicas para beneficiarios Fonasa es de $\$ 2.987$ (13,89\%) y 
Tabla 2. Porcentaje del gasto en salud según tipo de gasto y sistema de salud

\begin{tabular}{|c|c|c|c|c|c|c|c|c|}
\hline $\begin{array}{l}\text { Tipo de gasto/ } \\
\text { Sistema de salud }\end{array}$ & $\begin{array}{c}\text { Fonasa } \\
\text { A }\end{array}$ & $\begin{array}{c}\text { Fonasa } \\
\text { B }\end{array}$ & $\begin{array}{c}\text { Fonasa } \\
\mathrm{C}\end{array}$ & $\begin{array}{c}\text { Fonasa } \\
\text { D }\end{array}$ & $\begin{array}{l}\text { Isapre } \\
\text { abierta }\end{array}$ & $\begin{array}{l}\text { Isapre } \\
\text { cerrada }\end{array}$ & $\begin{array}{l}\text { Capredena/ } \\
\text { Dipreca }\end{array}$ & Otros \\
\hline Total de hogares & $8,19 \%$ & $26,06 \%$ & $19,82 \%$ & $12,13 \%$ & $29,79 \%$ & $1,26 \%$ & $2,31 \%$ & $0,44 \%$ \\
\hline Gasto en medicamentos & $40,03 \%$ & $30,75 \%$ & $31,39 \%$ & $30,46 \%$ & $30,21 \%$ & $22,91 \%$ & $41,99 \%$ & $61,61 \%$ \\
\hline Gasto en insumos médicos & $3,59 \%$ & $3,22 \%$ & $2,08 \%$ & $1,68 \%$ & $1,47 \%$ & $0,63 \%$ & $1,65 \%$ & $0,58 \%$ \\
\hline Gasto en lentes ópticos & $13,07 \%$ & $10,50 \%$ & $11,40 \%$ & $11,01 \%$ & $6,50 \%$ & $9,85 \%$ & $14,62 \%$ & $9,47 \%$ \\
\hline $\begin{array}{l}\text { Gasto en aparatos ortopé- } \\
\text { dicos y terapéuticos }\end{array}$ & $23,09 \%$ & $0,11 \%$ & $0,14 \%$ & $0,32 \%$ & $0,88 \%$ & $0,00 \%$ & $4,96 \%$ & $6,97 \%$ \\
\hline Gasto en consulta médica & $7,23 \%$ & $15,40 \%$ & $15,35 \%$ & $11,77 \%$ & $17,74 \%$ & $16,01 \%$ & $12,14 \%$ & $17,70 \%$ \\
\hline $\begin{array}{l}\text { Gasto en intervención } \\
\text { médica en operaciones }\end{array}$ & $0,00 \%$ & $0,02 \%$ & $0,16 \%$ & $0,33 \%$ & $1,22 \%$ & $0,00 \%$ & $0,00 \%$ & $0,00 \%$ \\
\hline $\begin{array}{l}\text { Gasto en honorarios de } \\
\text { otros profesionales }\end{array}$ & $0,00 \%$ & $1,36 \%$ & $0,56 \%$ & $1,79 \%$ & $1,90 \%$ & $1,99 \%$ & $0,25 \%$ & $0,00 \%$ \\
\hline Gasto en servicio dental & $0,00 \%$ & $17,43 \%$ & $14,39 \%$ & $15,50 \%$ & $14,22 \%$ & $5,34 \%$ & $3,73 \%$ & $0,00 \%$ \\
\hline $\begin{array}{l}\text { Gasto en análisis y servicio } \\
\text { de laboratorio y rayos }\end{array}$ & $0,00 \%$ & $9,91 \%$ & $9,39 \%$ & $8,80 \%$ & $7,39 \%$ & $16,62 \%$ & $9,29 \%$ & $0,93 \%$ \\
\hline Gasto en hospitalización & $7,22 \%$ & $10,94 \%$ & $15,07 \%$ & $18,11 \%$ & $17,28 \%$ & $26,63 \%$ & $7,70 \%$ & $1,78 \%$ \\
\hline $\begin{array}{l}\text { Gasto en servicio de emer- } \\
\text { gencia móvil }\end{array}$ & $5,77 \%$ & $0,09 \%$ & $0,00 \%$ & $0,07 \%$ & $0,38 \%$ & $0,00 \%$ & $0,22 \%$ & $0,97 \%$ \\
\hline Gasto en seguro de salud & $0,00 \%$ & $0,25 \%$ & $0,07 \%$ & $0,15 \%$ & $0,82 \%$ & $0,00 \%$ & $3,47 \%$ & $0,00 \%$ \\
\hline Total & $100,00 \%$ & $100,00 \%$ & $100,00 \%$ & $100,00 \%$ & $100,00 \%$ & $100,00 \%$ & $100,00 \%$ & $100,00 \%$ \\
\hline
\end{tabular}

Tabla 3. Gasto promedio mensual en salud según tipo de gasto y sistema de salud (datos agregados) (\$ abril de 2007)

\begin{tabular}{|lccccc|}
\hline Tipo de gasto/Sistema de salud & Total & Fonasa & Isapre & $\begin{array}{c}\text { Relación } \\
\text { Isapre/Fonasa Fonasa/Isapre }\end{array}$ & $\begin{array}{c}\text { Relación } \\
\text { Total de hogares }\end{array}$ \\
\hline Gasto en medicamentos & $1,381,809$ & 914,826 & 429,083 & 0.47 & 2.13 \\
\hline Gasto en insumos médicos & $13,014.97$ & $6,740.74$ & $25,837.62$ & 3.83 & 0.26 \\
\hline Gasto en lentes ópticos & 754.54 & 530.44 & $1,243.74$ & 2.34 & 0.43 \\
\hline Gasto en aparatos ortopédicos y terapéuticos & $3,510.41$ & $2,375.47$ & $5,723.38$ & 2.41 & 0.42 \\
\hline Gasto en consulta médica & 491.58 & 307.84 & 727.57 & 2.36 & 0.42 \\
\hline Gasto en intervención médica en operaciones & $6,868.52$ & $2,987.06$ & $15,258.93$ & 5.11 & 0.20 \\
\hline Gasto en honorarios de otros profesionales & 335.83 & 32.02 & $1,013.23$ & 31.65 & 0.03 \\
\hline Gasto en servicio dental & 679.68 & 253.35 & $1,640.99$ & 6.48 & 0.15 \\
\hline Gasto en análisis y servicio de laboratorio y rayos & $3,432.42$ & $1,913.24$ & $6,678.75$ & 3.49 & 0.27 \\
\hline Gasto en hospitalización & $6,799.89$ & $3,011.11$ & $15,226.64$ & 5.06 & 0.29 \\
\hline Gasto en servicio de emergencia móvil & 153.90 & 79.02 & 314.26 & 3.98 & 0.20 \\
\hline Gasto en seguro de salud & 267.31 & 33.58 & 681.03 & 20.28 & 0.25 \\
\hline Total & $42,207.34$ & $21,498.07$ & $86,328.93$ & 4.02 & 0.05 \\
\hline
\end{tabular}


Caracterización del gasto de bolsillo en salud en Chile - C. Castillo-Laborde et al

Tabla 4. Porcentaje de gasto en salud según tipo de gasto y sistema de salud (datos agregados)

\begin{tabular}{|lrrrcc|}
\hline Tipo de gasto/Sistema de salud & Total & Fonasa & Isapre & $\begin{array}{c}\text { Relación } \\
\text { Isapre/Fonasa Fonasa/lsapre }\end{array}$ & $\begin{array}{c}\text { Relación } \\
\text { Total de hogares }\end{array}$ \\
\hline Gasto en medicamentos & $100,00 \%$ & $66,20 \%$ & $31,05 \%$ & 0,47 & 2,13 \\
\hline Gasto en insumos médicos & $30,84 \%$ & $31,36 \%$ & $29,93 \%$ & 0,95 & 1,05 \\
\hline Gasto en lentes ópticos & $1,79 \%$ & $2,47 \%$ & $1,44 \%$ & 0,58 & 1,71 \\
\hline Gasto en aparatos ortopédicos y terapéuticos & $1,16 \%$ & $1,43 \%$ & $0,84 \%$ & 0,59 & 1,67 \\
\hline Gasto en consulta médica & $16,27 \%$ & $13,89 \%$ & $17,68 \%$ & 1,27 & 1,70 \\
\hline Gasto en intervención médica en operaciones & $0,80 \%$ & $0,15 \%$ & $1,17 \%$ & 7,88 & 0,79 \\
\hline Gasto en honorarios de otros profesionales & $1,61 \%$ & $1,18 \%$ & $1,90 \%$ & 1,61 & 0,13 \\
\hline Gasto en servicio dental & $13,97 \%$ & $15,04 \%$ & $13,88 \%$ & 0,92 & 1,08 \\
\hline Gasto en análisis y servicio de laboratorio y rayos & $8,13 \%$ & $8,90 \%$ & $7,74 \%$ & 0,87 & 1,15 \\
\hline Gasto en hospitalización & $16,11 \%$ & $14,01 \%$ & $17,64 \%$ & 1,26 & 0,79 \\
\hline Gasto en servicio de emergencia móvil & $0,36 \%$ & $0,37 \%$ & $0,36 \%$ & 0,99 & 1,01 \\
\hline Gasto en seguro de salud & $0,63 \%$ & $0,16 \%$ & $0,79 \%$ & 5,05 & 0,20 \\
\hline Total & $100,00 \%$ & $100,00 \%$ & $100,00 \%$ & 1,00 & 1,00 \\
\hline
\end{tabular}

para Isapre de $\$ 15.258(17,68 \%)$, mientras que el gasto en hospitalizaciones ascendería a $\$ 3.011$ para Fonasa $(14,01 \%)$ y $\$ 15.226$ para Isapre $(17,64 \%)$. Por último, en el caso de los servicios dentales, el gasto promedio para beneficiarios del Fonasa es de $\$ 3.234(15,04 \%)$ y para los beneficiarios de Isapre de $\$ 11.982(13,88 \%)$.

Como se mencionó en la sección de Material y Método, otro nivel de análisis está relacionado con la proporción que el gasto de bolsillo en salud representa del ingreso total de los hogares. En este contexto, cuando se considera a todos los hogares de la muestra, esta proporción asciende a $5 \%$, siendo de un 3,8\% para los hogares beneficiarios de Fonasa y un 6,1\% para los beneficiarios de Isapre.

En la Figura 1, cuando se analizan dichas proporciones en términos de los deciles intragrupos (calculados al interior de los grupos Fonasa e Isapre por separado), es interesante constatar que en el caso de los beneficiarios Isapre son los deciles de menores ingresos aquellos que dedican mayor porcentaje de ingreso a realizar pagos directos en salud (9,3\%), mientras que los deciles de mayores ingresos sólo destinan un 3,8\% a estos fines. Lo anterior contrasta con los resultados para los beneficiarios Fonasa, en que la proporción del ingreso de los hogares dedicada a pagos directos en salud se mantiene cercana al $4 \%$ para la mayoría de los deciles, siendo más baja para los deciles de menores ingresos.

Por último, se analizan las proporciones de gasto de bolsillo en términos de los deciles de ingreso a nivel nacional (Figura 2). Si bien al calcular utilizando la muestra completa de hogares, los hogares Isapre quedan sub-representados en los deciles bajos de ingreso y sobre-representados en los más altos, este análisis permite identificar hogares similares en términos de ingreso y compararlos directamente. Los datos muestran que el gasto de bolsillo como proporción del ingreso no cambian radicalmente para el grupo de beneficiarios Fonasa, manteniéndose en torno al $4 \%$ para la mayoría de los deciles. Sin embargo, cuando se revisan las proporciones asociadas a los beneficiarios Isapre, se observa que los beneficiarios pertenecientes a los deciles 2, 3 y 5 destinan una proporción de su ingreso (cercana al 10\%) a pagos directos en salud. Un dato interesante de resaltar es que los beneficiarios del decil 10 pertenecientes al Fonasa son aquellos que menor proporción de sus ingresos destinan a pagos directos en salud (2,2\%), siendo también en el caso de las Isapre uno de los gastos de bolsillo menores $(4,7 \%)$. 

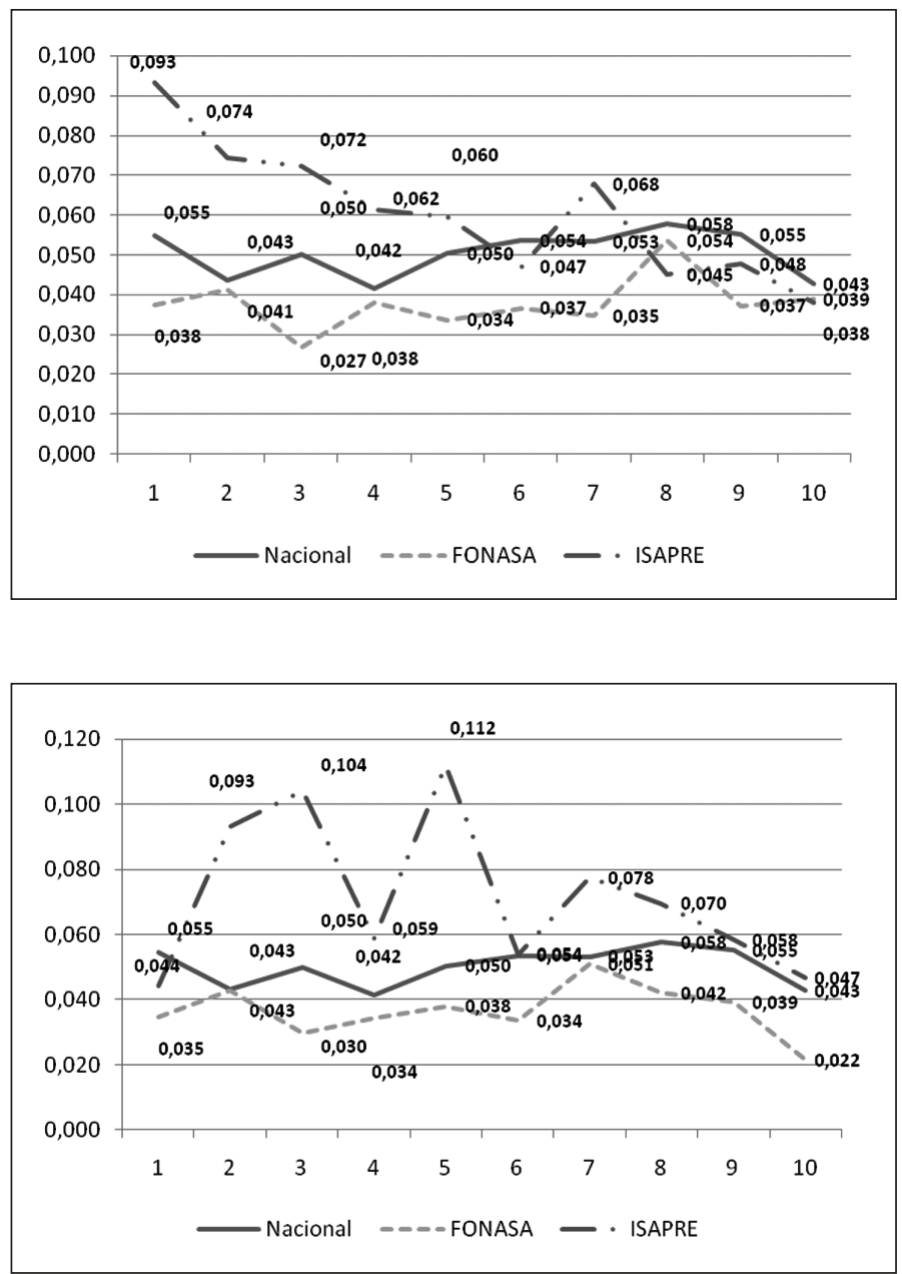

Figura 1. Relación gasto de bolsillo/ingreso (usando deciles intragrupos).
Figura 2. Relación gasto de bolsillo/ingreso (usando deciles a nivel nacional).

\section{Discusión}

El artículo presenta un análisis del gasto de bolsillo en salud, tanto en términos de su distribución entre los distintos ítems que lo conforman, como en términos de la proporción que representan respecto de los ingresos del hogar, explorando por primera vez las diferencias entre los hogares beneficiarios del Fonasa y las Isapre, e incluso de manera más desagregada, las diferencias entre los beneficiarios de los distintos grupos de Fonasa y distintos tipos de Isapre.

En términos generales, se constata la alta proporción de su ingreso que los hogares dedican a salud en forma de gasto de bolsillo en nuestro país, proporción que en el contexto internacional resulta alta tanto en comparación con los países de la $\mathrm{OECD}^{5}$, como con países de Latinoamérica ${ }^{6}$. De esta forma, se reitera la importancia de avanzar hacia una mayor protección financiera, que disminuya el gasto en salud realizado directamente del bolsillo de las personas.

En el caso de Chile, interesa además conocer si esta proporción es distinta dependiendo de si los hogares son beneficiarios de Fonasa o Isapre. Los resultados presentados muestran que los beneficiarios Isapre no sólo dedican más recursos para pagar directamente por salud en términos absolutos (cuatro veces el gasto de los beneficiarios Fonasa), sino que además gastan más en términos relativos dedicando $6,1 \%$ de sus ingresos a dichos fines (beneficiarios Fonasa dedican 3,8\%). En relación a la desagregación por tramos de Fonasa, resulta interesante constatar 
la gradiente socioeconómica inversa del gasto de bolsillo, siendo éste menor, en términos relativos y absolutos, para los beneficiarios de los tramos de menores ingresos.

En cuanto a la distribución del gasto para ambos sistemas también se presentan importantes diferencias: mientras el porcentaje de gasto en medicamentos es mayor para el tramo A de Fonasa y va disminuyendo gradualmente para los tramos de mayores ingresos, siendo cercano al de las Isapre abiertas para los beneficiarios del grupo $\mathrm{D}$, otros ítems como las consultas y las hospitalizaciones muestran la tendencia inversa, siendo menor la proporción para el tramos A y aumentando para los tramos de mayores ingresos y las Isapre abiertas. Lo anterior representa importante evidencia respecto de la protección financiera del seguro público en cuanto a atención institucional, pero también resalta la necesidad de avanzar a mayores coberturas en medicamentos.

Por otra parte, del análisis por deciles de ingreso intra-grupos, resulta interesante constatar que entre los hogares beneficiarios Isapre, son los más pobres quienes dedican una mayor proporción de su ingreso a pagos directos por salud. Por otra parte, del análisis por deciles a nivel nacional, se constata que para hogares en el mismo decil de ingreso, es decir, para hogares comparables en términos de ingreso, aquellos que pertenecen al sistema Isapre dedican un porcentaje mayor de su ingreso a pagar directamente por salud.

El análisis aquí presentado muestra importante evidencia respecto de la mayor protección financiera otorgada por el seguro público a sus beneficiarios. Al ser un análisis descriptivo, puede ser considerado una primera aproximación al análisis de las diferencias, en términos de protección financiera, entre el Fonasa y las Isapre, el cual se espera pueda ser complementado en el futuro por análisis estadísticos más complejos.

Agradecimientos: Los autores agradecen a Charles Durán del Dpto. de Investigación y Desa- rrollo del Instituto Nacional de Estadísticas, por la preparación de la base de datos solicitada a través de la página web institucional. Además se agradecen los comentarios de dos revisores anónimos a una primera versión este artículo.

\section{Referencias}

1. World Health Organization-WHO (2000) The World Health Report 2000: health systems-improving performance. Chapter 2: How well do health systems perform? Geneva, World Health Organization.

2. Kutzin, J. (2001). A descriptive framework for countrylevel analysis of health care financing arrangements. Health Policy 56 (3): 171-203.

3. World Health Organization-WHO (2010). Health systems financing: the path to universal coverage. Geneva, World Health Organization.

4. Castillo-Laborde C, Miranda S, Palacios A. (2011). Gasto de Salud en Chile: Análisis comparativo. Economía y Salud, Boletín semestral, 5 (2): 13-22.

5. OECD. (2011). Health at a Glance 2011: OECD Indicators. OECD Publishing. [Disponible en: http://dx.doi. org/10.1787/health_glance-2011-en].

6. Knaul F, Wong R, Arreola-Ornelas H, Méndez O, Network on Health Financing and Social Protection in Latin America and the Caribbean (LANET). (2011). Household catastrophic health expenditures: A comparative analysis of twelve Latin America and Caribbean Countries. Salud Pública de México, 53 (sup. 2): S85S95.

7. INE. (2008). Resultados Encuesta de Presupuestos Familiares. Nov. 2006-oct. 2007. Instituto Nacional de Estadística. Presentación.

8. Cid C, Prieto L. (2012). El gasto de bolsillo en salud: el caso de Chile, 1997 y 2007. Revista Panamericana de Salud Pública, 31 (4): 310-316. Fonasa (2010). Estadísticas Fonasa 2010: Demografía. [Disponible en: http://www. fonasa.cl/wps/wcm/connect/03c92fb1-3e3d-4171-bcb515d69d868686/01+-Demograf \%C3\%ADa_pagina_ web_\%2822-08- 011\%29JAV.xls?MOD=AJPERES\&atta chment=true\&id $=13137881855371]$. 